\section{Alineación espontánea de los incisivos inferiores apiñados después de la extracción de los caninos temporales}

Spontaneous alignment of crowded inferior incisives after the extraction of temporary canines

\section{Resumen}

En la presente investigación se evaluó el comportamiento de los incisivos inferiores después de las extracciones de los caninos temporales inferiores. El estudio se realizó en la clínica integral del niño de la Facultad de Odontología de la Universidad Nacional Mayor de San Marcos. La muestra estuvo conformada por 10 pacientes niños de ambos sexos comprendidos entre los 8 y 9 años de edad que gozaban de buena salud general y portadores de una maloclusión de Clase I con apiñamiento severo a nivel de los incisivos inferiores. Previamente se tomaron radiografías cefalométricas y panorámicas así como modelos de estudio para hacer el diagnóstico ortodóncico y seleccionar los casos. Se tomaron modelos de estudio 3 meses después de realizadas las extracciones de los caninos temporales para evaluar los cambios producidos a nivel de la zona anteroinferior; dando como resultado: una completa corrección del apiñamiento dentario inferior en un 50 \% de los casos, una corrección parcial en el 40 \% y ninguna corrección en el 10 \%.

\section{Tomás Oriel Orellana Manrique ${ }^{1}$, Iván Calderón Cortez ${ }^{2}$, Martín Orellana Manrique $^{3}$}

Departamento Académico de Estomatología Pediátrica.

Estudiante de pre grado.

Estudiante de Maestría.

1-3 Facultad de Odontología UNMSM. Lima Perú.

Correspondencia:

Mg. Tomás Oriel Orellana Manrique

Av. Diez Canseco 150-304.

Miraflores. Lima-Perú.

e-mail: torellanam@unmsm.edu.pe

Palabras Clave: Extracciones seriadas. Apiñamiento incisivo inferior. Caninos temporales.

\begin{abstract}
This investigation evaluated the behavior of permanent inferior incisives after the primary canine's extractions. The study was carried out in the Pediatric Clinic of Dental Faculty of San Marcos University. The sample was conformed by 10 patients, male and female children, between 8 and 9 years of age, with good general health, having Class I malocclusion and severe crowding of the inferior incisives. It was taken Cephalometric and Panoramic radiographies, as well as models to make the orthodontic diagnoses and select the cases. To evaluate changes that took place in the area, models were prepared 3 months after extractions, having as a result: a complete correction of the inferior tooth crowding in $50 \%$ of the cases, a partial correction in $40 \%$ and any correction in $10 \%$.
\end{abstract}

Key words: Serial tooth extraction. Inferior incisive's crowding. Primary canine.

\section{Introducción}

La presencia de los apiñamientos dentarios en los niños de corta edad es uno de los principales motivos de la consulta odontológica. El profesional odontólogo debe desde ese momento establecer un programa encaminado a solucionar este problema.

Estudios realizados por muchos investigadores han demostrado que el factor principal en la instalación de estos apiñamientos es el hereditario, ${ }^{(1)}$ debido principalmente al tamaño dentario. ${ }^{(2)} \mathrm{El}$ aumento de los cruces genéticos puede explicar en parte el incremento del apiñamiento observado en los últimos tiempos. Parece haber un fuerte control genético sobre las dimensiones de los maxilares y las dimensiones transversales e las arcadas que influyen directamente en la cantidad de espacio disponible para los dientes permanentes. Sin embargo no se puede dejar de mencionar la acción que ejerce el ambiente en el crecimiento. ${ }^{(3)}$ Las fuerzas producidas por los labios, las mejillas la lengua, los dedos y otros objetos pueden también influenciar en la posición final de los dientes. ${ }^{(4)}$

Una presunción diagnóstica de las anomalías de posición dentaria que se podrían instalar en el futuro se debería hacer cuando el niño tiene apenas 4 o 5 años de edad. Si a esta edad hay ausencia de los diastemas fisiológicos de crecimiento, tendremos casi la se- guridad que los incisivos permanentes no tendrán espacio para su adecuada alineación dentro de las arcadas dentarias. Estudios realizados en niños de 5 años de edad, encontraron que el espaciamiento incisivo en dentición temporal estaba presente solo en el $44,25 \%$.de la población estudiada. ${ }^{(5,6)}$

El apiñamiento dentario se instala desde que hacen su erupción los incisivos inferiores. En algunos casos la pérdida prematura de los caninos caducos provee el espacio suficiente para el buen alineamiento de los incisivos. Es así como a partir de estas observaciones Hotz en 1970, estableció la técnica de extracciones seriadas tratando de imitar lo que la naturaleza había proyectado ${ }^{(7,8)}$. 
Las extracciones seriadas esta recomendadas principalmente para pacientes que presentan maloclusiones de clase I con severa discrepancia alveolodentaria, es decir, una discrepancia entre la extensión del arco y el tamaño del diente ${ }^{(9)}$, puestos que en estos pacientes no existen problemas esqueléticas y la función muscular no se encuentra alterada.

La mejor época para iniciar la extracción seriada es cuando han hecho erupción los cuatro incisivos inferiores y cuando están erupcionando los incisivos laterales; esto es, cuando el niño ha cumplido los 8 o 9 años de edad, previo a un análisis radiográfico y del espacio.

La secuencia que se sigue en las extracciones seriadas será primero con la extacción de los caninos temporales, seguida posteriormente en el momento indicado con la extracción de los primeros molares temporales, para finalizar con la extracción de los primeros premolares.

Existen muchas razones para empezar un tratamiento temprano de las maloclusiones sobre todo con técnicas que minimizan los tratamientos con aparatología ortodóncica ${ }^{(10)}$. Sin embargo, tenemos que precisar que no se trata de una simple receta para ser aplicada a este tipo de maloclusión sino que va más allá, y para esto tenemos que hacer un buen diagnóstico, a fin de determinar si la discrepancia justifica el inicio de extracciones seriadas.

Con la extracción de los caninos temporales se pretende conseguir la corrección espontánea de las anomalías de posición de los incisivos por acción de los músculos de la lengua y de los labios, al haberse eliminados los problemas de espacio. $Y$ de esta manera se podría conseguir una mejor estabilidad en la corrección ortodóncica ${ }^{(11,12)}$

Algunos autores como Tweed preconizan los tratamientos con extracciones dentarias a fin de favorecer la verticalización de los incisivos inferiores, ya que según este autor, los incisivos inferiores que tienen una angulación cercana a los 90 grados con el plano mandibular presentan una mayor estabilidad después de la corrección ortodóncica $^{(13)}$.

En el presente estudio se inició la técnica de extracciones seriadas de caninos caducos inferiores a fin de observar los cambios de posición que tuvieron los incisivos inferiores apiñados al cabo de tres meses.

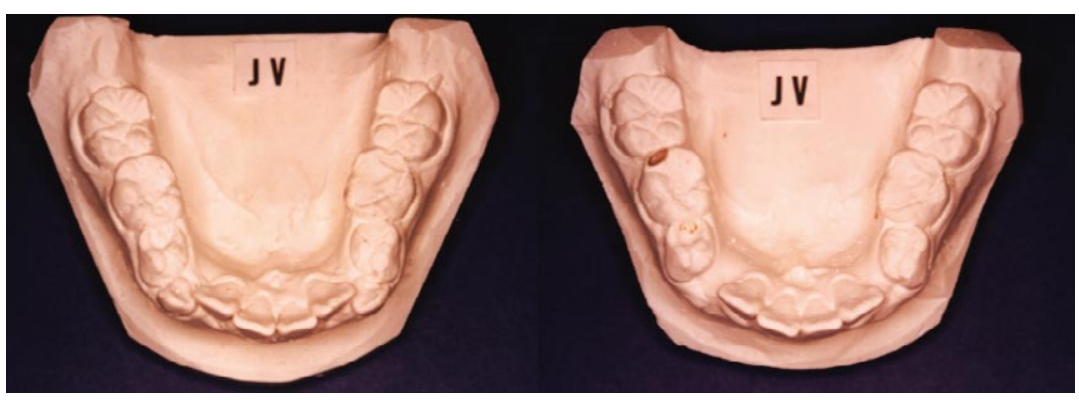

Fig. 1 Izquierda: modelos de estudio antes de la extracción de caninos temporales. Nótese el marcado apiñamiento dentario. Derecha: modelos de estudio 3 meses después de las extracciones. Se observa una completa corrección espontánea del apiñamiento dentario

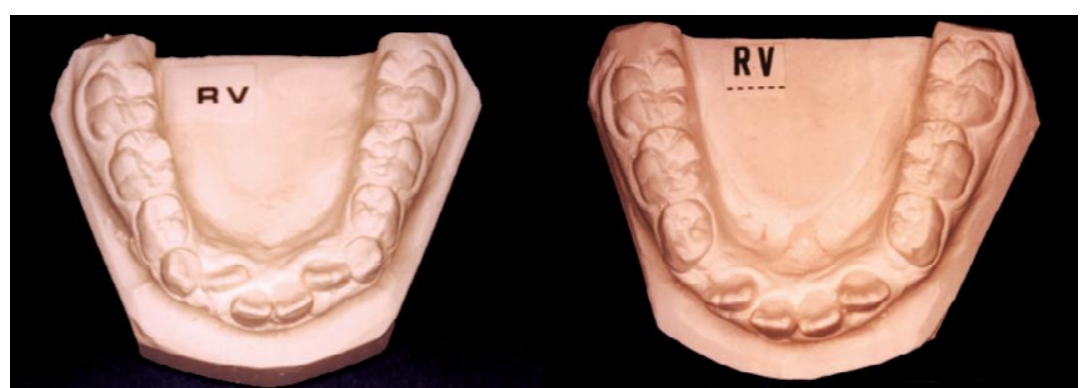

Fig 2 Izquierda: modelos de estudio de un caso con apiñamiento incisivo inferior con algun grado de rotación de los incisivos laterales. Derecha: modelos de estudio 3 meses después de las extracciones seriadas. Se nota una parcial corrección del apiñamiento dentario.

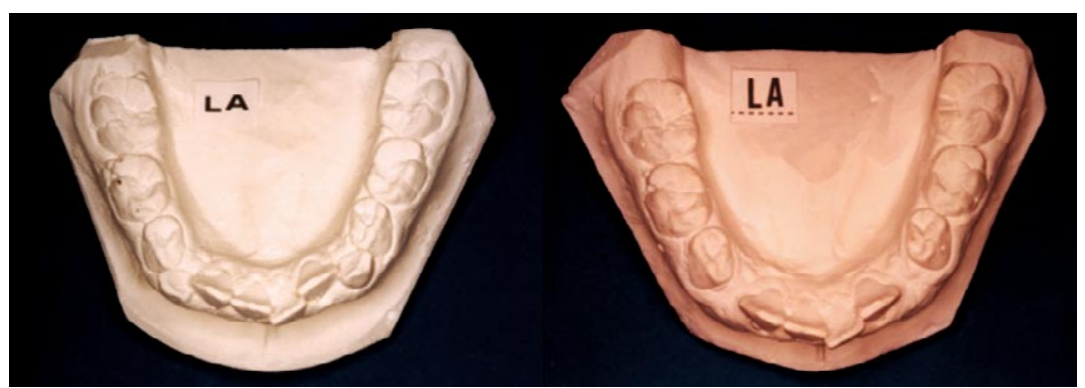

Fig 3 Izquierda: modelos de estudio antes de la extracción de caninos temporales con cierto grado de rotación del incisivo central inferior izquierdo. Derecha: modelos de estudio 3 meses después de la extracción de los caninos temporales. No se produjo corrección espontánea del apiñamiento dentario

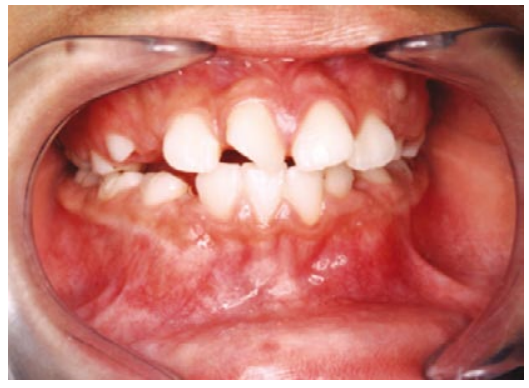

Fig 4 Vista intrabucal del caso en que no se produjo corrección espontánea del apiñamiento incisivo inferior. Se observa una mala articulación dentaria del sector anterior debido a la rotación que presentan los incisivos superiores.

La muestra estuvo constituida por 10 pacientes niños de ambos sexos, comprendidos entre 8 y 9 años de edad, que gozaban de una buena salud general, portadores de una maloclusión de Clase I, con apiñamiento severo de los incisivos inferiores y en los que se encontraban presentes los caninos temoporales de ambos lados. Dichos pacientes acudieron en busca de tratamiento ortodóncico a la Clínica Odontológica de la Facultad de Odontología de la Universidad Nacional Mayor de San Marcos.

Antes de aplicar la técnica de extracciones seriadas se tomaron los modelos de estudio y las radiografías cefalométricas y panorámicas para hacer el diagnóstico de la maloclusión. Trascurridos los tres meses después de las extracciones de los caninos temporales de ambos lados, se procedió a la toma de nuevos modelos de estudio y fotografías para evaluar los cambios producidos en la posición de los incisivos inferiores.

\section{Resultados}

Después de transcurridos tres meses de haber realizado las extracciones seriadas de los caninos temporales 
inferiores de ambos lados, se pudo evaluar en los modelos de estudio, los resultados obtenidos a nivel de los incisivos inferiores. En 5 casos (50\%) se observó una completa corrección espontánea del apiñamiento incisivo, en 4 casos la corrección fue parcial (40 $\%)$ y en 1 caso (10\%) no se produjo ninguna corrección.

\section{Discusión}

Antes de su erupción, los incisivos permanentes inferiores se encuentran en una posición lingualizada respecto a sus homólogos temporales y es durante su erupción que van adoptando una posición más vestibulizada ${ }^{(14,15)}$ En el caso de apiñamiento, los incisivos centrales se acomodarán primero dentro de la arcada y serán los incisivos laterales que al no encontrar el espacio suficiente quedarán lingualizados, hasta que se le de el espacio suficiente mediante las extracciones de los caninos temporales, siempre y cuando la función muscular de la lengua y los labios no se encuentre alterada ${ }^{(16)}$. Esto es lo que sucedió en el $50 \%$ de los casos estudiados en los cuales hubo una buena corrección espontánea del apiñamiento incisivo inferior (Fig. 1). En los casos en que se produjo una corrección parcial, los dientes apiñados se encontraban con algún grado de rotación, posición difícil de ser corregida por la acción conjunta del labio y de la lengua (Fig. 2). Al estudiar detenidamente, el caso en que no hubo corrección espontánea, se pudo observar la existencia de un marcado apiñamiento en los incisivos superiores por lo que posiblemente las malas relaciones en la oclusión no permitieron el movimiento dentario a nivel de los incisivos inferiores.(Fig. 4)

\section{Conclusiones}

1. La técnica de extracciones seriadas de caninos temporales inferiores induce satisfactoriamente hacia la corrección espontánea de los apiñamientos de los incisivos inferiores.

2. La técnica de extracciones seriadas de caninos caducos inferiores debe ser aplicada en los casos de maloclusión de Clase I.

3. Antes de iniciar un programa de extracciones seriadas de caninos temporales inferiores se deberán considerar algunos factores como la edad del paciente, el tipo de mordida, las rotaciones dentarias y la función muscular.

\section{Referencias bibliográficas}

1. Graber T.M. Ortodoncia Teoría y Práctica. 3a ed. México: Nueva Editorial Latinoamericana. 1974

2. Orellana O., Mendoza J., Perales S., Marengo H. Factores etiológicos de las maloclusiones en pacientes que acuden a tratamiento en la clínica integral del niño. Rev Odontología Sanmarquina 2001; Vol 1 (8)

3. Moss M.L., Salentijn L. The primary role of funtional matrices in facial growth. Am J Orthodontics; Vol 55 (6): 566-577

4. Moyers R. Manual de Ortodoncia. 1a ed. Buenos Aires: Editorial Mundi S.A.I.C. y F. 1976

5. Cepero A.S., Diaz R.J., García N., Blanco A.M. Estudio de la dentición temporal en niños de 5 años de edad. Rev Cubana de Ortodoncia, Julio-diciembre 1995

6. Ponce M., Hernández Y. Frecuencia y distribución de maloclusión en una población de 0 a 6 años de edad en San Luis de Potosí. México. Programa Bebé
Clínica Potosina. Rev Latinoamericana de Ortodoncia y Odontopediatría. 2006

7. Hotz R., Guidance of eruption versus serial extraction. Am J Orthodontics 1970; Vol 58 (1)

8. Dale J. A. A propósito de las extracciones seriadas. Rev D'Orthopedie DentoFaciale. Juillet 1978; (3)

9. Chaconas J. Ortodoncia. USA: El Manual Moderno. 1980

10. Graber T.M., Swain B. Ortodoncia Principios Generales y Técnicas. 2a ed. Argentina: Editorial Médica Panamericana. 1989

11.Nanda R., Burstone Ch. Contención y Estabilidad en Ortodoncia. Argentina. Editorial Médica Panamericana S.A. 1994

12. Moreno M.A., Muelas L., AlioJ.J. Factores que favorecen la aparición de recidivas tras el tratamiento ortodóncico. Rev de Actualidad Odonto-Estomatológica Española. Enero-Febrero. 1993

13. Tweed Ch. The Francfort Mandibular Plane Angle (FMIA) in Orthodontic Diagnosis, Treatment, Planning and Prognosis. Angle Orthodontic 1954; Vol 24: 121-169

14.Van Der Linden F. De la denture temporaire a la denture permanent. Rev D'Orthopedie Dento-Faciale 1979; Vol 13

15. Viazis A. Atlas de Ortodoncia, Principios y Aplicaciones Clínicas. Argentina: Editorial Médica Panamericana. 1995

16. Profit W. Ortodoncia, Teoría y Práctica. 2a ed. España: Editorial Mosby Doyma Libros S.A.1995

Recibido: 02-07-2008

Aceptado para publicación: 25-08-2008 